\title{
Posterior Reversible Encephalopathy Syndrome in pre-eclampsia presenting as Anton syndrome
}

\author{
${ }^{*}$ CG Hewavitharane ${ }^{1}$, EM Manoj ${ }^{2}$, DS Perera ${ }^{3}, R U$ Wanigasuriya $^{l}$, S Jayasinghe ${ }^{4}$, H Liyanage ${ }^{4}$ \\ Registrar ${ }^{1}$, Senior Registrar ${ }^{3}$ in Anaesthesiology, Senior Registrar in Critical Care Medicine ${ }^{2}$ \\ National Hospital of Sri Lanka, Sri Lanka, Consultant Anaesthetist ${ }^{4}$, De Zoysa Hospital for Women, \\ Sri Lanka.
}

*Corresponding author: chinxhewavitharane@gmail.com

\begin{abstract}
Introduction:
Posterior reversible encephalopathy syndrome (PRES) is a transient clinical neuroradiological entity caused by reversible ischemia most commonly of the posterior cerebral vasculature. Diagnosis ofPRES might be obscured when the patient presents with visual anasognosia (Anton syndrome) as the initial manifestation.
\end{abstract}

\section{Case report:}

An 18 year old lady presented with vomiting, severe headache and oedema at 32 weeks gestation. She had severe-preeclampsia evidenced by blood pressure of $160 / 110 \mathrm{mmHg}$, hyperreflexia and albuminuria. She further had visual anasognosia (cortical blindness) and a post-partum convulsion prompting the consideration of PRES, which was later confirmed by Magnetic Resonance imaging. She underwent immediate delivery by caesarean section followed by rapid reduction of blood pressure resulting in complete recovery within 48 hours.

\section{Conclusion:}

Prompt recognition of Posterior Reversible Encephalopathy Syndrome (PRES), which has a strong association with pre-eclampsia, is extremely important to prevent the associated morbidity and mortality. Eventhough, the vigilant management of PRES during ante-partum period might be complicated, this case consolidates the fact that early diagnosis of PRES leads to a favourable outcome.

Keywords:

Posterior reversible encephalopathy syndrome; visual anasognosia; Anton syndrome; preeclampsia

\section{Introduction}

Albeit a rare condition, Posterior Reversible Encephalopathy Syndrome (PRES) is most commonly reported in association with preeclampsia and eclampsia. ${ }^{1}$ In the acute setting, prompt recognition of PRES and the immediate treatment of the inciting condition is crucial to avoid the permanent damage leading to sequelae and even mortality during pregnancy.

Initial presentation of cortical blindness due to PRES with visual anosognosia (Anton syndrome) may readily be overlooked as the visual disturbances in severe pre-eclampsia. Even though PRES is increasingly recognized and reported in the current literature, its initial presentation as Anton syndrome is rarely reported. In this report, we present the successful management of an ante-partum pre-eclamptic case complicated by visual Anosognosia secondary to PRES followed by post-partum seizures.

\section{Case Presentation}

A 18 years old married ladyfrom a poor socioeconomic background, arrived to the hospital with vomiting, severe headache, facial puffiness and bilateral ankle oedema at 32 weeks gestation of her $2^{\text {nd }}$ pregnancy. On admission, she had walked into objects but denied of being blind with a Glasgow Coma Scale score of 14 out of 15. Her initial blood pressure recording was 160/110 $\mathrm{mmHg}$ with severe albuminuria and generalised hyperreflexia.Her lung bases were clear and urine production was adequate. She was treated with intravenous hydralazine and magnesium sulphate as for severe pre-eclampsia.

After 4 hours, she was admitted to the intensive care unit for further management as her blood 
pressure gradually rose to $200 / 130 \mathrm{mmHg}$ despite being on intravenous anti-hypertensives. She never had fever or seizures during the antepartum period, or a significant past medical history such as epilepsy, hypertension or connective tissue disorders. On asking her to describe the object in front of her, her reply was incorrect. Probable diagnosis of PRES was first suspected at this point considering the visual anasognosia associated with elevated blood pressure. Patient was oriented to time, place and person; speech, intelligence was normal but irritable in her behaviour. There was no abnormality of any other cranial nerve. Pupillary reflexes were intact with normal optic fundus. Patient had normal power in all four limbs and no sensory loss. Cerebellar functions were normal. There was no neck stiffness. Skull and spine examination was normal.

Her laboratory investigations revealed normal complete blood counts, renal function tests, serum electrolytes, liver enzymes and coagulation profile. She underwent emergency lower segment caesarean section under subarachnoid block and delivered a live baby. Postoperatively, 5 hours later she developed an episode of generalised tonic clonic seizure which was controlled by escalating the magnesium sulphate infusion. Her blood pressure was controlled by using labetalol infusion. Magnetic resonant imaging of the brain showed ill-defined areas of low signal intensity in T1- weighted images and abnormal high signal intensity in T2- weighted lesions distributed asymmetrically in the cerebral hemisphere bilaterally and left side of the brain stem. These findings were compatible with the clinical diagnosis of Posterior Reversible Encephalopathy Syndrome. (Figures 1and 2)

Figure 1

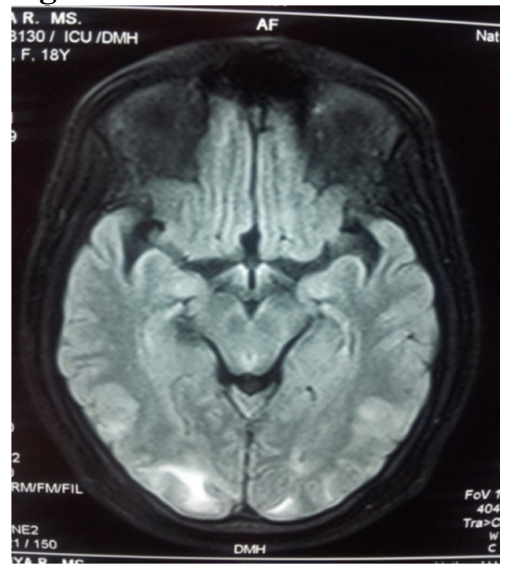

Figure 2

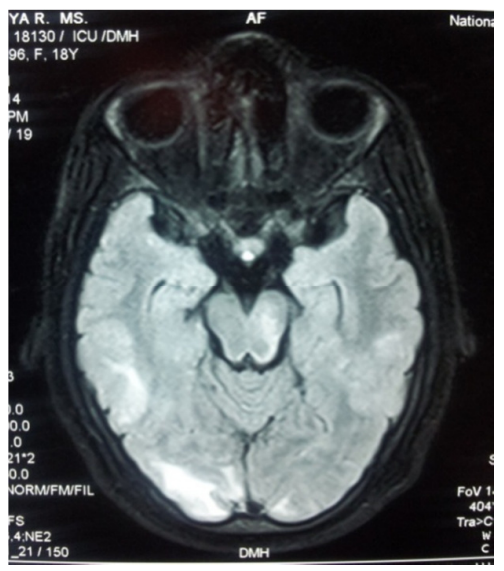

Both Magnetic Resonance Arteriogram and Venogram of the brain were normal with no evidence of cerebral vasoconstriction or cerebral venous sinus thrombosis. After 48 hours her vision completely recovered. She was discharged on oral nifedipine on the sixth postpartum day.

\section{Discussion}

An association between eclampsia and PRES was first described by Hinchey et al in $1996 .^{2}$ PRES, as the name suggests, is a constellation of symptoms (headache, altered consciousness, visual abnormalities, and seizures) caused by reversible ischaemia most commonly of the posterior cerebral vasculature, thus affecting the parietal- occipital region. PRES has been accompanied by a number of medical conditions such as hypertensive encephalopathy, preeclampsia, eclampsia, renal diseases, haemolytic uraemic syndrome, cytotoxic and immunosuppressant drugs, blood transfusions, and electrolyte disturbances. ${ }^{3}$

Initial presentation of this patient as visual anosognosiais a rare neurological scenario related to cortical blindness. ${ }^{4}$ In such a case, the anterior visual tracts are intact, but the visual association centres in the occipital cortex may be compromised.These patients deny their blindness and affirm adamantly that they are capable of seeing. The clinical presentation includes confabulations and sometimes confusional states.Convulsions are a common manifestation of both eclampsia as well as PRES. So the distinction of the cause of seizure in our patient during the immediate post-partum period is difficult. Since PRES is a completely reversible hypertensive emergency in the vast 
majority, we should be aware of this unusual association to reduce the morbidity and mortality.

Sibley et al reported a typical case of PRES in a Sri Lankan lady with pregnancy induced hypertension in puerperium, in which the patient was a suitable candidate for a rapid reduction of the blood pressure. ${ }^{5}$ However,the atypical presentation of PRES with Anton syndrome during the antepartum period might not only delay the diagnosis but complicate the drastic reduction of blood pressure which is the mainstay in management of this transient phenomenon. Therefore, this case highlights the importance of the low threshold to suspect this rare neurological association in order to achieve a favourable outcome.

\section{References}

1. Legriel S, Pico F, and Azoulay E. Understanding Posterior Reversible Encephalopathy Syndrome Annual Update in Intensive Care and Emergency Medicine 2011; 26: 833.
2. Hinchey J, Chaves C, Appignani B, et al. A reversible posterior leukoencephalopathy syndrome. N Engl J Med 1996; 334: 494-500. http://dx.doi.org/10.1056/NEJM1996022233408 $\underline{03}$ PMid:8559202

3. Pedraza R, Marik PE, Varon J. Posterior Reversible Encephalopathy Syndrome: A Review. Crit Care \& Shock 2009; 12:135-143.

4. Longo DL, Kasper DL, Jameson JL. Harrison's Principles of Internal Medicine, 18th edition, Vol. 2. The McGraw-Hill Companies: 370: $3287 \mathrm{p}$.

5. Sibly AJM, Caldera HPMC, Gamage R. Posterior Reversible Encephalopathy Syndrome in a patient with pregnancy induced hypertension in pueperium. Galle Medical Journal 2012;17: $1 ; 55-57$.

http://dx.doi.org/10.4038/gmj.v17i1.4364 